\title{
The TREND Statement
}

\author{
Elizabeth Treasure \\ Department of Dental Health and Biological Sciences, The Dental School, University of Wales College of \\ Medicine, Cardiff
}

Evidence-Based Dentistry (2004) 5, 88-91. doi:10.1038/sj.ebd.6400290

Readers will be familiar with the CONSORT guidelines ${ }^{1}$ for the reporting of randomised controlled trials. These guidelines were designed to improve the reporting of RCTs and are of considerable use to researchers designing and reporting studies as well as to editors and to systematic reviewers. However, a major limitation of CONSORT is that they are limited to RCTs and there are far more research designs than RCTs. Some topics just cannot be investigated using RCTs and yet it may often be desirable to try to identify whether or not there is a causal link between two factors. It is therefore very welcome to see the development of the TREND statement (www.trend-statement.org/asp/trend.asp) - the Transparent Reporting of Evaluations with NonRandomised designs. ${ }^{2}$ As the authors state these guidelines are developing there is room for alteration and improvement but their prime focus is to encourage the transparency or clarity of reporting.

In view of the evolutionary state of these guidelines, I would encourage researchers, editors and reviewers to become familiar with them and to start using them. As they are used, areas, primarily of omission, will become apparent. The first area that needs expanding, in my opinion, is that more detail is required describing the characteristics of each population subgroup. I consider that more prominence of the potential for confounding factors needs to be acknowledged.

As CONSORT has helped both investigators and reviewers improve the quality of RCTs and systematic reviews there is the possibility for TREND to do the same

The TREND Checklist (Version 1.0) ${ }^{2}$

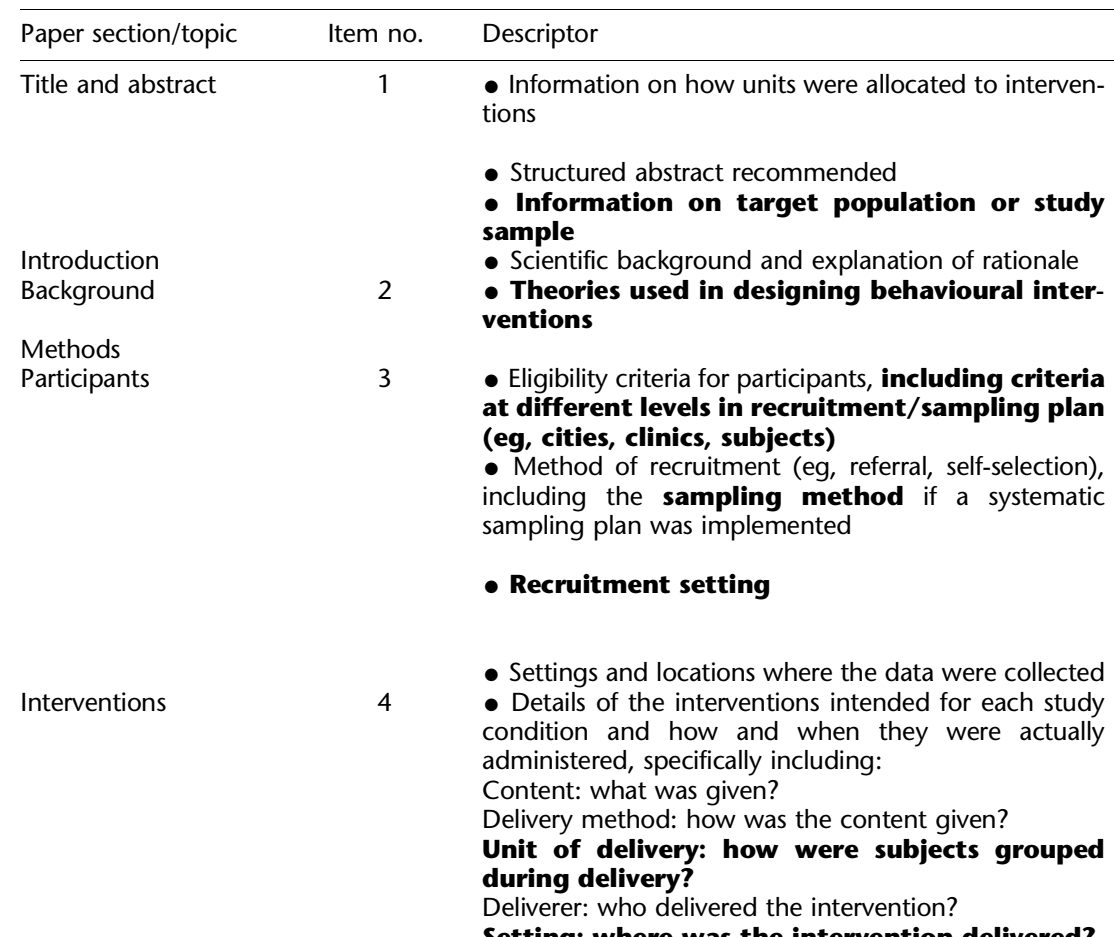

Setting: where was the intervention delivered?

Exposure quantity and duration: how many sessions or episodes or events were intended to be delivered? How long were they intended to last?
Examples from HIV behavioural prevention research

Example (title): A non-randomised trial of a clinic-based HIV counselling intervention for African American female drug users

Example (theory used): the community-based AIDS intervention was based on social learning theory

Example (sampling method): using an alphanumeric sorted list of possible venues and times for identifying eligible subjects, every tenth venue - time unit was selected for the location and timing of recruitment Examples (recruitment setting): subjects were approached by peer opinion leaders during conversations at gay bars Interventions

Example (unit of delivery): the intervention was delivered to small groups of 5-8 subjects

Examples (setting): the intervention was delivered in the bars; the intervention was delivered in the waiting rooms of sexually transmitted disease clinics

Examples (exposure quantity and duration): the intervention was delivered in five 1-h sessions; the intervention consisted of standard HIV counselling and testing (pretest and post-test counselling sessions, each about $30 \mathrm{~min}$ ) 


\begin{tabular}{|c|c|c|}
\hline Paper section/topic & Item no. & Descriptor \\
\hline & & $\begin{array}{l}\text { Time span: how long was it intended to take to deliver } \\
\text { the intervention to each unit? }\end{array}$ \\
\hline & & $\begin{array}{l}\text { Activities to increase compliance or adherence } \\
\text { (eg, incentives) }\end{array}$ \\
\hline Objectives & 5 & - Specific objectives and hypotheses \\
\hline \multirow[t]{2}{*}{ Outcomes } & 6 & $\begin{array}{l}\text { - Clearly defined primary and secondary outcome } \\
\text { measures } \\
\text { - Methods used to collect data and any methods } \\
\text { used to enhance the quality of measurements }\end{array}$ \\
\hline & & $\begin{array}{l}\text { - Information on validated instruments such as } \\
\text { psychometric and biometric properties }\end{array}$ \\
\hline Sample size & 7 & $\begin{array}{l}\text { - How sample size was determined and, when } \\
\text { applicable, explanation of any interim analyses and } \\
\text { stopping rules }\end{array}$ \\
\hline $\begin{array}{l}\text { Assignment } \\
\text { method }\end{array}$ & 8 & $\begin{array}{l}\text { - Unit of assignment (the unit being assigned } \\
\text { to study condition, eg, individual, group, } \\
\text { community) }\end{array}$ \\
\hline
\end{tabular}

- Method used to assign units to study conditions, including details of any restriction (eg, blocking, stratification, minimization)

- Inclusion of aspects employed to help minimise potential bias induced due to non-randomisation (eg, matching)

Blinding (masking)

Unit of analysis

Results

Participant flow

- If the unit of analysis differs from the unit of assignment, the analytical method used to account for this (eg, adjusting the standard error estimates by the design effect or using multilevel analysis)

- Statistical methods used to compare study groups for primary outcome(s), including complex methods for correlated data

- Statistical methods used for additional analyses, such as subgroup analyses and adjusted analysis

- Methods for imputing missing data, if used

- Statistical software or programs used
Examples from HIV behavioural prevention research

Examples (time span): each intervention session was to be delivered (in five 1-h sessions) once a week for 5 weeks; the intervention was to be delivered over a 1 -month period.

Example (activities to increase compliance or adherence): bus tokens and food stamps were provided

Examples (method used to collect data): self-report of behavioural data using a face-to-face interviewer-administered questionnaire; audio-computer-assisted selfadministered instrument

Example 1 (assignment method): subjects were assigned to study conditions using an alternating sequence wherein every other individual enrolled (eg, 1, 3, 5, etc) was assigned to the intervention condition and the alternate subjects enrolled (eg, 2, 4, 6, etc) were assigned to the comparison condition

Example 2 (assignment method): for odd weeks (eg 1, 3 , 5), subjects attending the clinic on Monday, Wednesday, and Friday were assigned to the intervention condition and those attending the clinic on Tuesday and Thursday were assigned to the comparison condition; this assignment was reversed for even weeks

Example (blinding): the staff member performing the assessments was not involved in implementing any aspect of the intervention and knew the participants only by their study identifier number

Example 1 (unit of analysis): since groups of individuals were assigned to study conditions, the analyses were performed at the group level, where mixed effects models were used to account for random subject effects within each group

Example 2 (unit of analysis): since analyses were performed at the individual level and communities were randomised, a prior estimate of the intraclass correlation coefficient was used to adjust the standard error estimates before calculating confidence intervals 


\begin{tabular}{lcl} 
Paper section/topic & Item no. & Descriptor \\
\hline Recruitment & 13 & $\begin{array}{l}\text { - Dates defining the periods of recruitment and } \\
\text { follow-up } \\
\text { - Baseline demographic and clinical characteristics of } \\
\text { participants in each study condition }\end{array}$ \\
Baseline data & 14 &
\end{tabular}

- Baseline characteristics for each study condition relevant to specific disease prevention research

- Baseline comparisons of those lost to followup and those retained, overall and by the study condition

- Comparison between the study population at baseline and the target population of interest

Baseline equivalence

- Data on the study group equivalence at baseline and statistical methods used to control for baseline differences
Numbers

Outcomes and

estimation

Ancillary analyses

Adverse events

Discussion

Interpretation

7

- For each primary and secondary outcome, a summary of results for each study condition, and the estimated effect size and a confidence interval to indicate the precision

- Inclusion of null and negative findings

- Inclusion of results from testing prespecified causal pathways through which the intervention was intended to operate, if any

- Summary of other analyses performed, including subgroup or restricted analyses, indicating which are prespecified or exploratory

- Summary of all important adverse events or unintended effects in each condition (including summary measures, effect size estimates, and confidence intervals)

- Interpretation of the results, taking into account study hypotheses, sources of potential bias, imprecision of measures, multiplicative analyses, and other limitations or weaknesses of the study

- Discussion of results taking into account the mechanism by which the intervention was intended to work (causal pathways) or alternative mechanisms or explanations

- Discussion of the success of and barriers to implementing the intervention, fidelity of implementation

- Discussion of research, programmatic, or policy implications

Generalizability

- Generalizability (external validity) of the trial findings, taking into account the study population, the characteristics of the intervention, length of follow-up, incentives, compliance rates, specific sites/settings involved in the study, and other contextual issues

Overall evidence
Examples from HIV behavioural prevention research

Example (baseline characteristics specific to HIV prevention research): HIV serostatus and HIV testing behaviour

Example (baseline equivalence): the intervention and comparison groups did not statistically differ with respect to demographic data (gender, age, race/ethnicity; $P>0.05$ for each),but the intervention group reported a significantly greater baseline frequency of injection drug use $(P=0.03)$; all regression analyses included baseline frequency of injection drug use as a covariate in the model

Example (number of participants included in the analysis): the analysis of condom use analysed included only those who reported at the 6-month follow-up having had vaginal or anal sex in the past 3 months (75/125 for intervention group and 35/60 for standard group)

Example ("intention to treat"): the primary analysis was intention to treat and included all subjects as assigned with available 9-month outcome data (125 of 176 assigned to the intervention and 110 of 164 assigned to the standard condition)

Example (ancillary analyses): although the study was not powered for this hypothesis an exploratory analysis shows that the intervention effect was greater among women than among men (although not statistically significant)

Example (adverse events): police cracked down on prostitution, which drove the target study population, commercial sex workers, to areas outside the recruitment/ sampling area

Note. Masking (blinding) of participants or those administering the intervention may not be relevant or possible for many behavioural interventions. Theories used to design the interventions (see item 2) could also be reported as part of item 4. The comparison between study population at baseline and target population of interest (see item 14) could also be reported as part of item 21.

Descriptors appearing in boldface are specifically added, modified, or further emphasised from the CONSORT statement. Boldface topic and descriptors are not included in the CONSORT statement but are relevant for behavioural interventions using nonrandomised experimental designs. The CONSORT statement ${ }^{1}$ or the explanation document for the CONSORT statement ${ }^{3}$ provides relevant examples for any topic or descriptor that is not in boldface. 
for non-randomised interventions. Who should then use TREND? Anyone designing a non-randomised intervention. However, if you are well into such an intervention it would be well worth your time revising the protocol against TREND to see if there are any more data that you need to collect. As CONSORT does for RCTs TREND will be particularly useful when it comes to writing up and reviewing these interventions. Editors should be encouraged to adopt TREND.
As someone who has systematically reviewed questions examined by RCTs and non-randomised interventions the process of finding the necessary information can be difficult. Although it is usual practice to contact authors it is rare to be given much additional useful information. TREND achieves a useful step forward in the reporting the of literature. Researchers and reviewers should be encouraged to use TREND which should improve the quality of work undertaken.
1. Moher D, Schulz KF, Altman DG. The CONSORT statement: revised recommendationsfor improving the quality of reports of parallel-group randomized trials. Lancet 2001; 357:1191-1194.

2. Des Jarlais DC, Lyles C, Crepaz N. TREND Group. Improving the reporting quality of nonrandomized evaluations of behavioral and public health interventions: the TREND statement. Am J Public Health 2004; 94:361-366.

3. Moher D, Jones A, Lepage L. Use of the CONSORT statement and quality of reports of randomized trials: a comparative before-and-after evaluation. JAMA 2001; 285:1992-1995. 